\title{
Probabilistic Fiber-Tracking Reveals Degeneration of the Contralateral Auditory Pathway in Patients with Vestibular Schwannoma
}

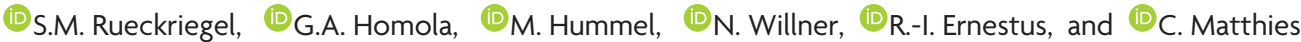

\begin{abstract}
BACKGROUND AND PURPOSE: Vestibular schwannomas cause progressive hearing loss by direct damage to the vestibulocochlear nerve. The cerebral mechanisms of degeneration or plasticity are not well-understood. Therefore, the goal of our study was to show the feasibility of probabilistic fiber-tracking of the auditory pathway in patients with vestibular schwannomas and to compare the ipsi- and contralateral volume and integrity, to test differences between the hemispheres.
\end{abstract}

MATERIALS AND METHODS: Fifteen patients with vestibular schwannomas were investigated before surgery. Diffusion-weighted imaging (25 directions) was performed on a 3T MR imaging system. Probabilistic tractography was performed for 3 partial sections of the auditory pathway. Volume and fractional anisotropy were determined and compared ipsilaterally and contralaterally. The laterality ratio was correlated with the level of hearing loss.

RESULTS: Anatomically reasonable tracts were depicted in all patients for the acoustic radiation. Volume was significantly decreased on the hemisphere contralateral to the tumor side for the acoustic radiation and diencephalic section, while fractional anisotropy did not differ significantly. Tracking did not yield meaningful tracts in 3 patients for the thalamocortical section and in 5 patients for the diencephalic section. No statistically significant correlations between the laterality quotient and classification of hearing loss were found.

CONCLUSIONS: For the first time, this study showed that different sections of the auditory pathway between the inferior colliculus and the auditory cortex can be visualized by using probabilistic tractography. A significant volume decrease of the auditory pathway on the contralateral hemisphere was observed and may be explained by transsynaptic degeneration of the crossing auditory pathway.

ABBREVIATIONS: $F A=$ fractional anisotropy; $I C=$ inferior colliculus; $M G N=$ medial geniculate nucleus

$V^{e}$ estibular schwannoma, also known as acoustic neurinoma, is a common intrameatal and intracranial tumor evolving from the eighth cranial nerve with an incidence of $10-15$ per million per year. ${ }^{1}$ The tumor usually arises from Schwann cells within the vestibular part of the eighth cranial nerve. The benign tumor is characterized by a slow growth pattern for years. ${ }^{2,3}$ The slow-butsteady increase in size causes progressive damage to the neurons of the eighth cranial nerve, leading to hearing impairment, tinni-

Received October 2, 2015; accepted after revision March 6, 2016.

From the Departments of Neurosurgery (S.M.R., M.H., N.W., R.-I.E., C.M.) and Neuroradiology (G.A.H.), Würzburg University Hospital, Würzburg, Germany.

A research grant as part of the "program of first grant application" was provided by the Interdisciplinary Center of Clinical Research, University Hospital Würzburg, to Stefan Rueckriegel.

Paper previously presented in part at: Annual Meeting of the German Society of Neurosurgery, June 7-10, 2015; Karlsruhe, Germany.

Please address correspondence to Stefan Rueckriegel, MD, Department of Neurosurgery, Würzburg University Hospital, Josef-Schneider-Str 11, 97080 Würzburg,

Germany; e-mail: rueckriege_s@ukw.de

http://dx.doi.org/10.3174/ajnr.A4833 tus, and vertigo. ${ }^{4-6}$ Typically the vertigo symptoms are transient, while hearing impairment can progressively deteriorate toward unilateral deafness ipsilateral to the lesion side. To estimate the extent of hearing loss, examination of tone audiometry and speech discrimination is crucial. ${ }^{6-9}$ Large tumors involving neighboring structures such as the fifth or seventh cranial nerve or even the brain stem and cerebellum may cause facial paresis and numbness, cerebellar ataxia, or corticospinal tract-related sensorimotor deficits. There are various classification systems, but tumor extension is usually classified on the basis of the size from T1 (intrameatal localization) to $\mathrm{T} 4$ (compression of the brain stem). ${ }^{10}$ Treatment options include surgical removal of the tumor and radiation therapy. During the past decades, the surgical goal has shifted from gross total resection toward optimal functional outcome. $^{8,11}$

Despite surgical tumor removal, improvement of hearing is unusual, even if anatomic preservation of the eighth cranial nerve has been achieved and resection has led to decompression of the nerve. Apparently, the cochlear nerve itself and possibly cortical 
and subcortical auditory structures have a low potential for regeneration after nerve damage. In contrast, the function of the damaged vestibular nerve is compensated by the integrating circuitry of the equilibrium: The latter involves the vestibular, visual, and somatosensory systems ${ }^{12}$ and therefore provides a more robust reserve for compensation. Although functional outcome has been the treatment focus, the interest was limited to the individual nerve structures, while the cerebral mechanisms of degeneration or plasticity of the associated white matter tracts and cortex areas are rarely investigated and understood.

The first studies by DTI, in unselected hearing disorders, succeeded, in some cases, in illustrating abnormality of white matter integrity of the auditory pathway. $\mathrm{Wu}$ et $\mathrm{al}^{13}$ found decreased fractional anisotropy (FA) at the contralateral inferior colliculus and lateral lemniscus in 19 patients with non-tumor-related sensorineural hearing loss. Chang et $\mathrm{al}^{14}$ reported abnormalities of fractional anisotropy in several parts of the auditory pathway when comparing 10 patients with sensorineural hearing loss with healthy subjects. Both investigations indicated a transsynaptic degeneration of the auditory pathway. An association between DTIderived measures and abnormalities in brain stem auditoryevoked potentials was illustrated in preterm infants. ${ }^{15}$ These previous investigations measured DTI-derived parameters like FA or radial diffusivity by using ROI analyses. While the feasibility of fiber-tracking has been shown previously in auditory pathway investigation, ${ }^{16-18}$ this method has not been hitherto applied to the auditory pathway in patients with vestibular schwannoma. A detailed analysis of the white matter microstructure of the auditory pathway and of possible abnormalities in patients with vestibular schwannoma does not exist, to our knowledge. Therefore, the primary goal of our study was to show the feasibility of probabilistic fiber-tracking of partial sections of the auditory pathway in patients with vestibular schwannomas and to quantify the volume and integrity of these sections ipsi- and contralateral to the tumor side to test for differences between the hemispheres. The secondary goal was to identify associations of the integrity of the auditory pathway with audiometric measurements, including speech discrimination and the electrophysiologic brain stem-evoked potentials.

\section{MATERIALS AND METHODS}

Fifteen patients ( 7 women, 8 men; mean age, $50.5 \pm 13.7$ years; demographic details in Table 1) with a unilateral vestibular schwannoma and a given indication to undergo tumor resection with a retrosigmoid approach were included in this pilot study. None of the patients were previously diagnosed with another disease of the central nervous system such as Alzheimer disease or multiple sclerosis. Patients underwent routine preoperative MR imaging, including contrast-enhanced T1-weighted imaging and CISS sequences. DTI and high-resolution 3D T1-weighted MPRAGE images were added to the preoperative routine imaging performed on a 3T scanner (Magnetom Trio; Siemens, Erlangen, Germany) with a 12-channel head coil. Two DTI datasets were obtained by using 25 directions (section thickness, $3.6 \mathrm{~mm}$; inplane resolution, $1.8 \times 1.8 \mathrm{~mm}^{2}$ ). Diffusion data were converted from DICOM to NIfTI format and further processed by using the
Table 1: Patient characteristics ${ }^{\text {a }}$

\begin{tabular}{lcccccc}
\hline Patient No. & Age $(\mathbf{y r})$ & Sex & T. Side & T. Size & AC & EC \\
\hline 1 & 64 & M & L & T3a & H1 & A4 \\
2 & 47 & M & R & T4b & H2 & A2 \\
3 & 44 & M & R & T3b & H3 & A3 \\
4 & 54 & F & L & T2a & H2 & A2 \\
5 & 50 & M & L & T3b & H2 & A2 \\
6 & 60 & F & L & T4a & H5 & A5 \\
7 & 57 & F & L & T3b & H6 & A4 \\
8 & 46 & F & L & T3a & H2 & A2 \\
9 & 58 & F & L & T3b & H4 & A3 \\
10 & 73 & F & R & T2a & H3 & A4 \\
11 & 43 & M & R & T2b & H4 & A3 \\
12 & 23 & M & R & T4b & H2 & A3 \\
13 & 42 & M & R & T4a & H2 & A2 \\
14 & 68 & M & L & T3a & H6 & A5 \\
15 & 29 & F & R & T4b & H6 & A5 \\
\hline
\end{tabular}

Note:-T. indicates tumor; AC, audiometric classification; EC, electrophysiologic classification based on auditory brain stem responses; L, left; R, right.

${ }^{a}$ Fifteen patients with vestibular schwannoma were included in the study. Tumor size was between $\mathrm{T} 2 \mathrm{a}$ and $\mathrm{T} 4 \mathrm{~b}$.

FMRIB Software Library (FSL, Version 5.0; http://www.fmrib.ox. ac.uk/fsl). ${ }^{19}$ After applying eddy current correction, brain extraction, and fitting the tensor models averaging the 2 datasets (DTIFit; http://fsl.fmrib.ox.ac.uk/fsl/fsl-4.1.9/fdt/fdt_dtifit.html), we further processed data by using bedpostX (http://fsl.fmrib.ox. ac.uk/fsl/fslwiki/FDT/UserGuide\#BEDPOSTX).

The high-resolution T1-weighted image was coregistered to the diffusion space to define the seed masks for fiber-tracking in diffusion space. We accepted the lower resolution of diffusion space so that seed volumes would not have to be coregistered subsequently to a different space, introducing spatial uncertainty for the tracking procedure. An overlay of the coregistered T1weighted and FA images was used to define the seed masks for the fiber-tracking process. Three partial sections of the auditory pathway were tracked by using 2 seed masks per section at the start and ending points of the tracts: Seed masks of the lateral section (Fig 1) were defined at the auditory cortex and Sylvian fissure. Seed masks of the long lateral section (Fig 2) were defined at the auditory cortex and the medial geniculate nucleus (MGN). Seed masks of the diencephalic section (Fig 3) were defined at the inferior colliculus and MGN. Each seed mask was assigned to a specific volume in all patients. Seed masks were defined on both hemispheres.

Probabilistic fiber-tracking experiments were performed for these 3 partial sections of the auditory pathway, with 6 fibertracking runs per patient by using probtrackx, part of FSL (http:// fsl.fmrib.ox.ac.uk/fsl/fslwiki/FDT/UserGuide). ${ }^{20-22}$ The "multiple masks" option of probtrackx was applied. A termination mask of fractional anisotropy $<0.1$ was used to avoid tracking throughout the cortex and fissures. The number of samples of probabilistic tracking was set at 5000, the curvature threshold was 0.2 , and a loop check was performed. The maximum number of steps was 2000 , and the step length was $0.5 \mathrm{~mm}$. The threshold of probability values was adjusted individually in the resulting volumes to exclude anatomically unreasonable voxels with low probability. The volume and FA of the results were determined. We did not determine further diffusivity indices because a higher number of tests would be statistically disadvantageous in the rather small sample size. FA and volume were compared intraindi- 


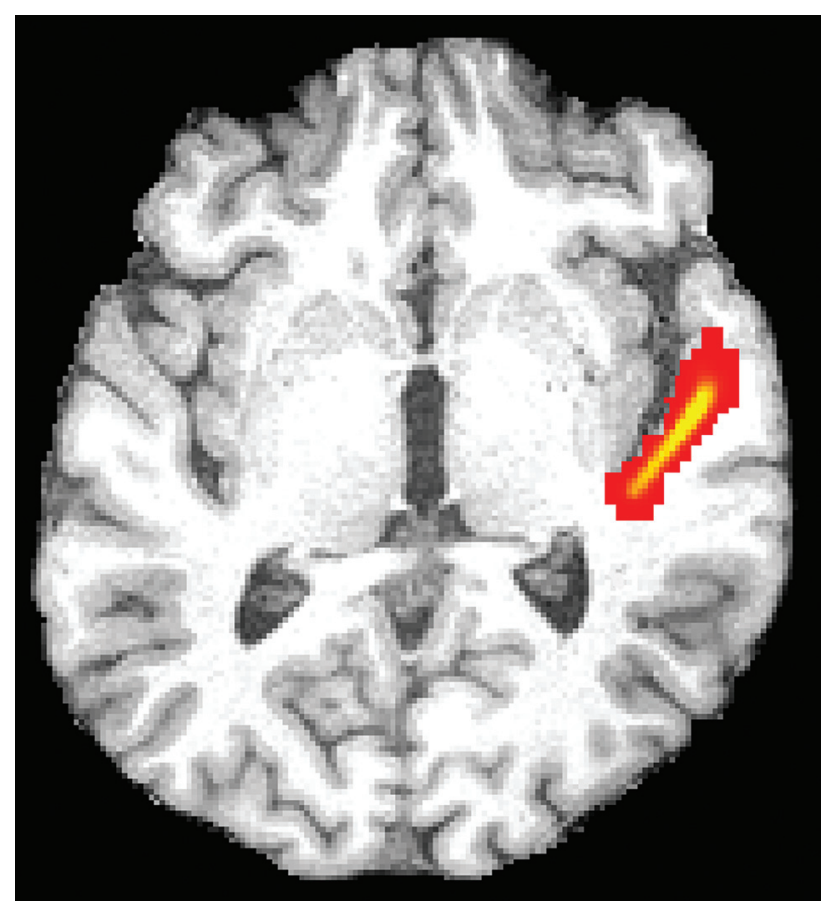

FIG 1. Illustration of the probabilistic fiber-tracking results of patient 3 of the left lateral section of the auditory pathway from the posteromedial Sylvian fissure to the primary auditory cortex on a representative axial section (red indicates low probability values; yellow, high probability values). Volume and mean fractional anisotropy of the 3D-depicted fiber tracts were determined ipsi- and contralateral to the tumor.

vidually between the ipsi- and contralateral hemisphere by using the Wilcoxon test. A laterality ratio was built by dividing the ipsilateral by the contralateral parameters. The laterality ratio was correlated with classifications of the audiometric and electrophysiologic (brain stem-evoked potentials) levels of hearing loss.

Speech discrimination and hearing function on both sides were tested preoperatively, and an audiometric classification was carried out according to the Hannover classification, ${ }^{7}$ which consists of 6 classes and is divided into $20-\mathrm{dB}$ steps. Class 1 is the best one and includes hearing loss up to $20 \mathrm{~dB}$ as an average in the 1 - to $3-\mathrm{kHz}$ zone and patients with a speech discrimination score of at least $95 \%$. The speech discrimination score is the percentage of words that were identified by the patient in the speech audiometry. Class 2 includes a 21 - to $40-\mathrm{dB}$ hearing loss and a minimum of a $70 \%$ speech discrimination score. Class 3 includes a 41 - to $60-\mathrm{dB}$ hearing loss and at least a $40 \%$ speech discrimination score. Class 4 includes 61 - to $80-\mathrm{dB}$ hearing loss and at least a $10 \%$ speech discrimination score. Class 5 is $>80-\mathrm{dB}$ hearing loss and a $0 \%-9 \%$ speech discrimination score. Class 6 is the worst class and includes $>100 \mathrm{~dB}$ hearing loss and a $0 \%$ speech discrimination score.

Auditory Brainstem Response was recorded by needle electrodes, and as a stimulus, we used a click that was applied by air-conducted earphones. Auditory Brainstem Response measurements were performed during the entire operation. To build an electropysiologic classification, the Auditory Brainstem Response quality at the beginning of surgery was categorized according to the Hannover classification and used for correlation. ${ }^{6}$ The

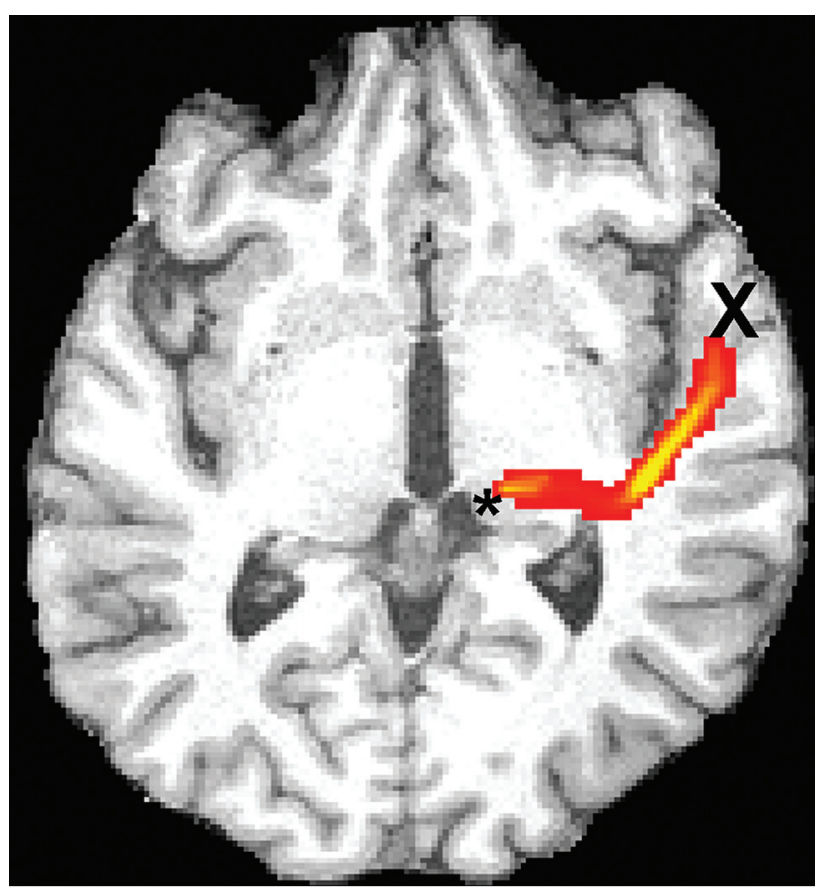

FIG 2. Illustration of the probabilistic fiber-tracking results of patient 3 of the left long lateral section of the auditory pathway from the medial geniculate nucleus (asterisk) to the primary auditory cortex (X) on a representative axial section. The tract crosses the external capsule and bends posteriorly along the Sylvian fissure, then anteriorly toward the primary auditory cortex.

Auditory Brainstem Response quality was categorized according to the presence of waves I, III, and V according the Hannover classification. Class 1, the best one, shows an Auditory Brainstem Response with waves I, III, and V at normal latency such as in healthy individuals; class 2 shows the same waves with pathologic latencies; in class 3, wave III is lost; in class 4, only wave I or wave V is reproducible; and in class 5, no reproducible waves exist.

The examiners who postprocessed the diffusion data were blinded to the clinical data of the patients.

The University Hospital Ethics Committee approved the study, and all patients gave informed consent.

\section{Statistical Analysis}

A paired group comparison between the ipsilateral and the contralateral parameters (volume and FA) of each section of the auditory pathway was performed by using the Wilcoxon signed rank test.

To perform a correlation analysis with functional parameters, we built the laterality quotient between ipsilateral and contralateral parameters (volume and FA) for each section of the auditory pathway. We used a Spearman correlation coefficient to assess the association between audiometric and electrophysiologic measurements and the laterality quotients of the auditory pathway volumes and FA. Furthermore, we used a Spearman correlation coefficient to assess the association between the size of the tumor according to the tumor-size classification (T2a-T3b, ordinal scale) and the laterality quotients of the auditory pathway volumes and FA. 


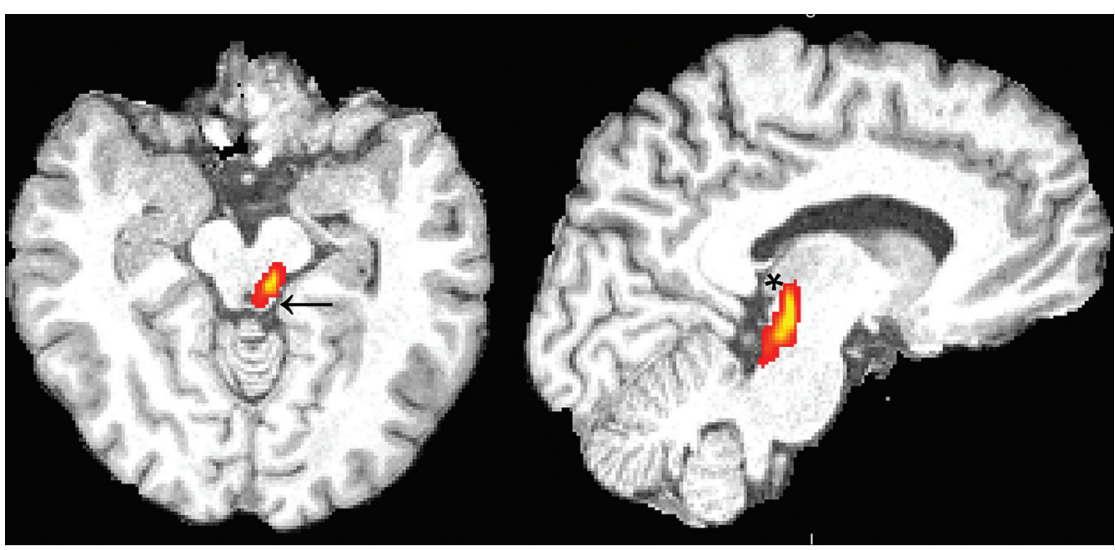

FIG 3. Illustration of the probabilistic fiber-tracking result of patient 4 of the left diencephalic section from the inferior colliculus (arrow) to the medial geniculate nucleus (asterisk) on sagittal and axial sections.

Table 2: Volume and FA of the ipsilateral and contralateral sections of the auditory pathway

\begin{tabular}{lcccccc}
\hline \multicolumn{1}{c}{ Section } & Volume II & Volume Cl & $\boldsymbol{P}$ & FA Il & FA Cl & $\boldsymbol{P}$ \\
\hline Lateral & $1957 \pm 668$ & $1480 \pm 516$ & $.017^{\mathrm{a}}$ & $0.38 \pm 0.04$ & $0.36 \pm 0.04$ & .72 \\
Long lateral & $1753 \pm 756$ & $1930 \pm 532$ & .314 & $0.44 \pm 0.04$ & $0.45 \pm 0.06$ & .72 \\
Diencephalic & $571.9 \pm 249$ & $410.7 \pm 390$ & $.009^{\mathrm{a}}$ & $0.31 \pm 0.08$ & $0.32 \pm 0.07$ & 1.00 \\
\hline
\end{tabular}

Note:- -1 indicates ipsilateral; $\mathrm{Cl}$, contralateral.

a $P<.05$.

Table 3: Correlations between laterality quotients of tracking parameters and audiometric or electrophysiologic classifications ${ }^{a}$

\begin{tabular}{llll}
\hline \multicolumn{1}{c}{ Laterality Quotient } & \multicolumn{1}{c}{ AC } & \multicolumn{1}{c}{ EC } & \multicolumn{1}{c}{ Tumor Size } \\
\hline Volume of lateral section & $r=-0.08, P=.78$ & $r=-0.08, P=.78$ & $r=0.19, P=.49$ \\
FA of lateral section & $r=0.14, P=.62$ & $r=0.14, P=.62$ & $r=-0.45, P=.09$ \\
Volume of long lateral section & $r=-0.12, P=.74$ & $r=-0.12, P=.74$ & $r=0.28, P=.42$ \\
FA of long lateral section & $r=0.46, P=.18$ & $r=0.46, P=.18$ & $r=0.03, P=.95$ \\
Volume of diencephalic section & $r=0.56, P=0.09$ & $r=0.56, P=.09$ & $r=-0.94, P=.80$ \\
FA of diencephalic section & $r=-0.40, P=.26$ & $r=0.40, P=.26$ & $r=0.20, P=.59$
\end{tabular}

Note:-AC indicates audiometric classification; EC, electrophysiologic classification based on auditory brain stem responses.

${ }^{a}$ No significant correlations were found between tracking parameters and audiometric measurements, electrophysiologic measurements, or tumor size (ordinal scale according to tumor classification T2a-T4b).

\section{RESULTS}

Anatomically reasonable tracts were depicted in all patients on both sides for the lateral section of the auditory pathway (Fig 1). Because this part of the auditory pathway was short and broad, high probability values were reached. The volume of the lateral section was significantly decreased on the hemisphere contralateral to the tumor side (mean volume ipsilateral, $1.957 \pm 0.668$; mean volume contralateral, $1.480 \pm 0.516 ; P=.017$; Table 2), while fractional anisotropy did not differ significantly.

It was possible to depict the long lateral section between the MGN and auditory cortex in most patients; the tracking result was a long, thin volume passing through the posterior limb of the internal capsule, the external capsule, and the corona radiata (Fig 2). Tracking did not yield any meaningful tracts in 3 patients for the long lateral section on both hemispheres and in 2 patients on 1 hemisphere. Therefore, the intraindividual comparison was possible with only 10 patients. No significant differences in volume or FA were found in the long lateral section (mean volume ipsilateral, $1.75 \pm 0.76$; mean volume contralateral, $1.225 \pm 0.53$; $P=.31)$.

The diencephalic section of the auditory pathway was identi- fied between the inferior colliculus (IC) and the ipsilateral MGN (Fig 3). It was not possible to track this section in $3 \mathrm{pa}$ tients on both sides and again in 2 patients on 1 side. Hence, intraindividual comparison was again possible with only 10 patients. Nevertheless, the volume of the contralateral diencephalic section was significantly decreased (mean volume ipsilateral, $0.572 \pm 0.249$; mean volume contralateral, $0.411 \pm$ $0.390 ; P=.009$ ), while fractional anisotropy did not differ.

No significant correlations between the laterality quotient and the audiometric and electrophysiologic classifications of hearing loss and tumor size were found (Table 3). Nevertheless, a trend toward associations between the laterality quotient of the diencephalic section volume of the auditory pathway and the electrophysiologic and audiometric quality classification by using the Hannover classification was detected (Table 3). A high laterality quotient was associated with impaired audiometric and electrophysiologic hearing function.

\section{DISCUSSION \\ Tractography of the Auditory Pathway}

This study shows, for the first time, that visualization of different sections of the auditory pathway between the IC and auditory cortex is feasible by using probabilistic tractography in an anatomically reasonable manner in patients with ves-

tibular schwannomas. There are several advantages of a tractography-based analysis of white matter tracts compared with ROIbased approaches. The anatomic course of the acoustic radiation is $3 \mathrm{D}$ and complex in shape, so the definition of the ROIs on axial 2D tensor maps would not be precise. Furthermore, ROI-based analyses do not comprise crossing fibers and their effect on fractional anisotropy, which is crucial given the anatomic course of the acoustic radiation crossing perpendicular fiber tracts such as the external capsule. In contrast, the fiber-tracking technology we used is capable of modeling crossing fibers and thereby minimizing their effects on the target parameters. Only a few previous publications have described tractography experiments of the auditory pathway itself. Keifer et $\mathrm{al}^{16}$ used the same methodology (probtrackx, FSL) and showed, in 13 healthy subjects, the connectivity of the MGN with the auditory cortex passing through the internal capsule, particularly the posterior limb, and crossing over the external capsule. This finding is the precondition to tracking the auditory pathway shown in our study. Profant et $\mathrm{al}^{17}$ also succeeded in tracking the auditory pathway by using probabilistic fiber-tracking in an investigation on aging in 54 subjects; how-

AJNR Am J Neuroradiol 37:1610-16 Sep 2016 www.ajnr.org 1613 
ever, only the index of longitudinal diffusivity trended toward positive correlation with increasing age. A direct tracking of the IC to the auditory cortex was performed, though passing through the MGN with low FA; therefore, a high uncertainty has to be assumed. Javad et $\mathrm{al}^{18}$ combined fMRI with hearing paradigms for sound, pitch, and melody and probabilistic fiber-tracking in 13 healthy subjects. The spatial tracking results from the activation areas to the IC were anatomically similar to our resulting volumes of the long lateral section.

\section{Degeneration of the Auditory Pathway}

We identified a significant decrease in volume of the lateral and diencephalic sections on the contralateral hemisphere. This decrease indicates a degeneration of the auditory pathway that is pronounced at the hemisphere contralateral to the lesion. Anatomic studies previously revealed the predominant projection of fiber tracts from the cochlear nucleus to the contralateral IC and MGN, while ipsilateral projections also exist. ${ }^{23,24}$

However, the comparison of the ipsi- and contralateral sides of the lesion might not fully represent the extent of degeneration because of bilaterally projecting fibers. Degeneration of bilateral fibers might result in decreased volumes of the auditory pathway on both hemispheres. The comparison of FA of the investigated sections of the auditory pathway did not reveal significant differences; this may also be attributed to degenerative effects of bilateral projecting fibers causing only nonsignificant side differences. Furthermore, previous anatomic and MR image studies indicated a structural difference between the dominant and the nondominant hemisphere per se, which is also true for the white matter of the superior temporal lobe. ${ }^{25,26}$

This structural difference between the dominant and nondominant hemispheres might further mask potential differences of FA not identified in our analysis. Kurtcan et $\mathrm{al}^{27}$ previously demonstrated a decrease of FA at the level of the IC by using an ROI analysis when comparing patients with vestibular schwannoma and healthy controls. This decrease was bilateral but stronger on the contralateral side; this finding supports the hypothesis that a bilateral degeneration with a stronger impact on the contralateral side occurred. However, a decrease of FA was not found at the levels of the MGN and auditory cortex in the analysis of Kurtcan et al. The ROI approach with a small transverse section of 4 pixels did not allow determination of a tract volume. Thus, it cannot be directly compared to our results. Furthermore, the determination of FA within the gray matter of the relay stations and cortex (IC, MGN, and auditory cortex) produces results with higher uncertainty because the baseline FA of the cortex and nuclear regions is low.

In the literature, there is no previous description of fiber tract integrity of the auditory pathway in patients with vestibular schwannomas, to our knowledge. However, some studies used DTI in patients with different disorders associated with hearing impairment. Especially patients with tinnitus were investigated by using DTI. ${ }^{28,29}$ Most interesting, probabilistic fiber-tracking in comparison with a healthy control group showed decreased FA of the connectivity between the auditory cortex and amygdala in patients with tinnitus. ${ }^{30}$ On the other hand, Husain et $\mathrm{al}^{31}$ found a stronger decrease of gray matter volume and FA in cerebral regions associated with hearing in patients with hearing loss without tinnitus than in those with tinnitus. Furthermore, previous studies by using ROI analyses of DTI indices found significant alterations of the auditory pathway in patients with congenital and traumatic sensorineural hearing loss. ${ }^{13,14,32,33}$ Another pilot study with a small number of patients focused on changes of the auditory nerve in patients with sensorineural hearing loss. ${ }^{34}$ This study showed significant changes of DTI metrics of the auditory nerve itself compared with healthy control subjects, suggesting a degeneration of the neuronal projections of the auditory nerve.

Our finding of decreased volume of contralateral sections of the auditory pathway might correspond to a transsynaptic degeneration of the crossing auditory pathway secondary to the damage of the ipsilateral cochlear nerve. A previous structural analysis of gray matter volume in 15 patients with vestibular schwannoma found a correlation between volume increase of the contralesional auditory cortex and stronger hearing impairment, ${ }^{35}$ which might be attributed to a compensatory allocation of the cortical field. Despite this volume increase of the cortical field, we found evidence of volume decrease of the auditory pathway conveying the auditory information to the contralesional cortex. Therefore, compensatory mechanisms seem to take place at the level of the cortical primary, projection, and association areas. Whether this compensatory mechanism is actually useful for the hearing function is a matter for future investigation. Most interesting, volume increase was also found bilaterally in the primary somatosensory cortices and the motion-sensitive cortex of the medial temporal gyrus. This may be linked to a multisensory compensation of the lesion and the vestibular system. However, the degeneration of the contralateral auditory tract that we showed in the current analysis might be a further reason, besides the lesion of the cochlear nerve itself, for the limited capacity of regaining hearing function after surgery in patients with vestibular schwannomas.

Although the audiometric and electrophysiologic classifications are also based on a functional deterioration by degeneration of the auditory pathway, we were not able to detect significant associations. Nevertheless, the laterality quotient of the tract volume of the diencephalic section of the auditory pathway tended to correlate with the audiometric and electrophysiologic classifications of hearing function. Most interesting, the electrophysiologic measurement included the fifth wave, which corresponds to a response of the inferior colliculus. The inferior colliculus was part of the diencephalic section of the auditory pathway that trended toward correlation. However, the missing detection of a significant association among morphologic tract integrity, hearing function, and tumor size might be due to the rather low patient number. One previous study reported a significant association between DTI-derived measures and abnormalities in the Auditory Brainstem Response in 56 preterm infants. ${ }^{15}$ Moreover, a variety of electrophysiologic measures were fed into the correlation analysis with DTI parameters.

\section{Limitations and Perspectives}

At present, the interpretation of our study results is restricted due to some limitations. The sample size of 15 patients is rather low; therefore, the statistical power of the tests performed is limited.

Because the diffusion tensor imaging was integrated into the 
standard preoperative MR imaging, the scanning time for the sequences was limited. Therefore, rather thick sections of $3.6 \mathrm{~mm}$ were chosen. Thirty-two directions are sufficient for the application of probabilistic fiber-tracking, yet a higher number of directions would have enhanced the quality of the data.

The distribution of tumor size is not representative because it did not contain any $\mathrm{T} 1$ tumors, and it did contain a high proportion, $80 \%$, of rather large tumors (T3a-T4b). Furthermore, the intraindividual comparison of fiber tracts cannot identify bilateral degeneration, but merely lateralized alterations. Patients with left- and right-sided tumors were included; thus, a possible effect of hemisphere dominance might confound the results. Hence, larger cross-sectional and longitudinal studies should be performed comparing changes of fiber tracts in patients with vestibular schwannoma versus healthy controls. Nonetheless, the identification of volume decrease contralateral to the tumor side provides an important finding for clinical discussion and decision-making: It explains, at least in part, why recovery of auditory function is rare and why auditory rehabilitation by hearing implants after tumor surgery is currently limited. ${ }^{36}$ Whether early tumor resection can prevent the described neurodegeneration will be a subject for future investigations.

\section{CONCLUSIONS}

This study shows, for the first time, that different sections of the auditory pathway between the inferior colliculus and the auditory cortex can be visualized by using probabilistic tractography. A significant volume decrease of the lateral and diencephalic sections on the contralateral hemisphere was observed and may be explained by transsynaptic degeneration of the crossing auditory pathway evolving from damage to the ipsilateral cochlear nerve. This might be a reason for the limited potential of hearing rehabilitation in these patients. Further longitudinal studies investigating changes of the auditory pathway and associated tracts with time are warranted for a better understanding of disease- and therapy-related degeneration and plasticity.

Disclosures: Stefan M. Rueckriegel, Maria Hummel, Ralf-Ingo Ernestus-RELATED: Grant: A grant of the Interdisziplinäres Zentrum für Klinische Forschung, University Hospital Würzburg, provided cofunding for a technician position. * Money paid to Institution.

\section{REFERENCES}

1. Howitz MF, Johansen C, Tos M, et al. Incidence of vestibular schwannoma in Denmark, 1977-1995. Am J Otol 2000;21:690-94 Medline

2. Bakkouri WE, Kania RE, Guichard JP, et al. Conservative management of 386 cases of unilateral vestibular schwannoma: tumor growth and consequences for treatment. J Neurosurg 2009;110: 662-69 CrossRef Medline

3. Hajioff D, Raut VV, Walsh RM, et al. Conservative management of vestibular schwannomas: third review of a 10-year prospective study. Clin Otolaryngol 2008;33:255-59 Medline

4. Axelsson A, Ringdahl A. Tinnitus: a study of its prevalence and characteristics. Br J Audiol 1989;23:53-62 Medline

5. Sughrue ME, Yang I, Aranda D, et al. The natural history of untreated sporadic vestibular schwannomas: a comprehensive review of hearing outcomes. J Neurosurg 2010;112:163-67 CrossRef Medline

6. Matthies C, Samii M. Management of vestibular schwannomas (acoustic neuromas): the value of neurophysiology for evaluation and prediction of auditory function in 420 cases. Neurosurgery 1997; 40:919-29; discussion 929-30 Medline

7. Samii M, Matthies C. Management of 1000 vestibular schwannomas (acoustic neuromas): hearing function in 1000 tumor resections. Neurosurgery 1997;40:248-60; discussion 260-62 Medline

8. Gardner G, Robertson JH. Hearing preservation in unilateral acoustic neuroma surgery. Ann Otol Rhinol Laryngol 1988;97:55-66 Medline

9. Samii M, Gerganov V, Samii A. Improved preservation of hearing and facial nerve function in vestibular schwannoma surgery via the retrosigmoid approach in a series of $\mathbf{2 0 0}$ patients. J Neurosurg 2006; 105:527-35 Medline

10. Sekiya T, Hatayama T, Shimamura N, et al. A comprehensive classification system of vestibular schwannomas. J Clin Neurosci 2000;7: 129-33 Medline

11. Akard W, Tubbs RS, Seymour ZA, et al. Evolution of techniques for the resection of vestibular schwannomas: from saving life to saving function. J Neurosurg 2009;110:642-47 CrossRef Medline

12. Bronstein AM. Vision and vertigo: some visual aspects of vestibular disorders. J Neurol 2004;251:381-87 Medline

13. Wu CM, Ng SH, Wang JJ, et al. Diffusion tensor imaging of the subcortical auditory tract in subjects with congenital cochlear nerve deficiency. AJNR Am J Neuroradiol 2009;30:1773-77 CrossRef Medline

14. Chang Y, Lee SH, Lee YJ, et al. Auditory neural pathway evaluation on sensorineural hearing loss using diffusion tensor imaging. $\mathrm{Neu}$ roreport 2004;15:1699-703 Medline

15. Reiman M, Parkkola R, Johansson R, et al. Diffusion tensor imaging of the inferior colliculus and brainstem auditory-evoked potentials in preterm infants. Pediatr Radiol 2009;39:804-09 CrossRef Medline

16. Keifer OP, Jr., Gutman DA, Hecht EE, et al. A comparative analysis of mouse and human medial geniculate nucleus connectivity: a DTI and anterograde tracing study. Neuroimage 2015;105:53-66 CrossRef Medline

17. Profant $\mathrm{O}$, Škoch A, Balogová Z, et al. Diffusion tensor imaging and MR morphometry of the central auditory pathway and auditory cortex in aging. Neuroscience 2014;260:87-97 CrossRef Medline

18. Javad F, Warren JD, Micallef C, et al. Auditory tracts identified with combined fMRI and diffusion tractography. Neuroimage 2014;84: 562-74 CrossRef Medline

19. Smith SM, Jenkinson M, Woolrich MW, et al. Advances in functional and structural MR image analysis and implementation as FSL. Neuroimage 2004;23(suppl 1):S208-19 Medline

20. Behrens TE, Berg HJ, Jbabdi S, et al. Probabilistic diffusion tractography with multiple fibre orientations: what can we gain? Neuroimage 2007;34:144-55 Medline

21. Behrens TE, Woolrich MW, Jenkinson M, et al. Characterization and propagation of uncertainty in diffusion-weighted MR imaging. Magn Reson Med 2003;50:1077-88 Medline

22. Jbabdi S, Sotiropoulos SN, Savio AM, et al. Model-based analysis of multishell diffusion MR data for tractography: how to get over fitting problems. Magn Reson Med 2012;68:1846-55 CrossRef Medline

23. Anderson LA, Malmierca MS, Wallace MN, et al. Evidence for a direct, short latency projection from the dorsal cochlear nucleus to the auditory thalamus in the guinea pig. Eur J Neurosci 2006;24: 491-98 Medline

24. Schofield BR, Motts SD, Mellott JG, et al. Projections from the dorsal and ventral cochlear nuclei to the medial geniculate body. Front Neuroanat 2014;8:10 CrossRef Medline

25. Vandermosten $M$, Poelmans $H$, Sunaert $S$, et al. White matter lateralization and interhemispheric coherence to auditory modulations in normal reading and dyslexic adults. Neuropsychologia 2013;51: 2087-99 CrossRef Medline

26. Anderson B, Southern BD, Powers RE. Anatomic asymmetries of the posterior superior temporal lobes: a postmortem study. Neuropsychiatry Neuropsychol Behav Neurol 1999;12:247-54 Medline

27. Kurtcan S, Alkan A, Kilicarslan R, et al. Auditory pathway features 
determined by DTI in subjects with unilateral acoustic neuroma. Clin Neuroradiol 2015 Mar 27. [Epub ahead of print] Medline

28. Lee YJ, Bae SJ, Lee SH, et al. Evaluation of white matter structures in patients with tinnitus using diffusion tensor imaging. J Clin Neurosci 2007;14:515-19 Medline

29. Aldhafeeri FM, Mackenzie I, Kay T, et al. Neuroanatomical correlates of tinnitus revealed by cortical thickness analysis and diffusion tensor imaging. Neuroradiology 2012;54:883-92 CrossRef Medline

30. Crippa A, Lanting CP, van Dijk P, et al. A diffusion tensor imaging study on the auditory system and tinnitus. Open Neuroimag J 2010; 4:16-25 CrossRef Medline

31. Husain FT, Medina RE, Davis CW, et al. Neuroanatomical changes due to hearing loss and chronic tinnitus: a combined VBM and DTI study. Brain Res 2011;1369:74-88 CrossRef Medline

32. Manners DN, Rizzo G, La Morgia C, et al. Diffusion tensor imaging mapping of brain white matter pathology in mitochondrial optic neuropathies. AJNR Am J Neuroradiol 2015;36:1259-65 CrossRef Medline

33. Lin Y, Wang J, Wu C, et al. Diffusion tensor imaging of the auditory pathway in sensorineural hearing loss: changes in radial diffusivity and diffusion anisotropy. J Magn Reson Imaging 2008;28:598-603 Medline

34. Vos SB, Haakma W, Versnel H, et al. Diffusion tensor imaging of the auditory nerve in patients with long-term single-sided deafness. Hear Res 2015;323:1-8 CrossRef Medline

35. Helmchen C, Klinkenstein JC, Krüger A, et al. Structural brain changes following peripheral vestibulo-cochlear lesion may indicate multisensory compensation. J Neurol Neurosurg Psychiatry 2011;82:309-16 CrossRef Medline

36. Merkus P, Di Lella F, Di Trapani G, et al. Indications and contraindications of auditory brainstem implants: systematic review and illustrative cases. Eur Arch Otorhinolaryngol 2014;271:3-13 CrossRef Medline 